\title{
Cervical cancer stage IIB-IVA radiation therapy clinical responses based on spectral pulsed wave Doppler
}

\author{
Syamel Muhammad, Dodi Suardi, Maringan DLT
}

Oncology Gynaecology Division of Andalas University Padang- Indonesia

Address: Syamel Muhammad: email: syamelmuhammad.sm@gmail.com ; phone: +628126648977

Medical Faculty of Andalas University Padang: Perintis kemerdekaan street Number.94

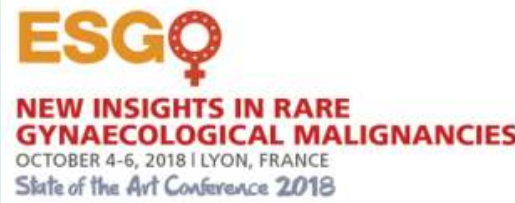

Objective: To examine the relationship between Doppler spectralultrasound images of Transrectal PulseWave Doppler with the clinical response of external radiation therapy for locally advance dcervical cancer (IIB-IVA). Method: Methods: The study used a prospective cohort study involving 60 samples of patients with stage IIB-IVA cervical cancer. Doppler Pulsewave checks use transrectal probes. Measurements of tumor mass and clinical assessment were performed before and after external radiation to assess the therapeutic response. Samples were grouped on Doppler pulsewave examinations into good and bad vascularizations which were then followed to assess the therapeutic response divided into good clinical response and poor clinical response.

Result: Of the 60 samples, 2 patients were unable to continue the study so that the clinical response was obtained as much as 46 people $(79.3 \%)$ and bad clinical response of 12 people (20.7\%). The group of bad clinical responses as much as $9(75.0 \%)$ had spectral vascularization poorly and as much as $3(25.0 \%)$ had good spectralvasculatory well while for good clinical response as much 19 (41.3\%) had spectral vascularization poor and as many as 27 (58, 7\%) have good spectral vascularization. In the analysis with Fisher exact test found a significant relationship $(p<0.05)$ between the spectral PW Doppler transrectal image with the clinical response of external radiation therapy in stage IIBIVA cervical cancer with RelativeRisk (RR) value 3,214 times.

\begin{tabular}{|l|c|c|c|} 
& \multicolumn{2}{|c|}{ Clinical respons } & $\left.\mathbf{p}^{*}\right)$ \\
\hline & $\begin{array}{c}\text { Bad } \\
(\mathbf{n = 1 2})\end{array}$ & $\begin{array}{c}\text { Good } \\
(\mathbf{n}=\mathbf{4 6})\end{array}$ & \\
\hline Spectral Doppler & 9 & 19 & $\mathrm{p}: 0.039$ \\
Bad & 3 & 27 & $\mathrm{RR} \mathrm{Cl}(95 \%): 3,214$ \\
Good & & & $(0,967-10.681)$
\end{tabular}
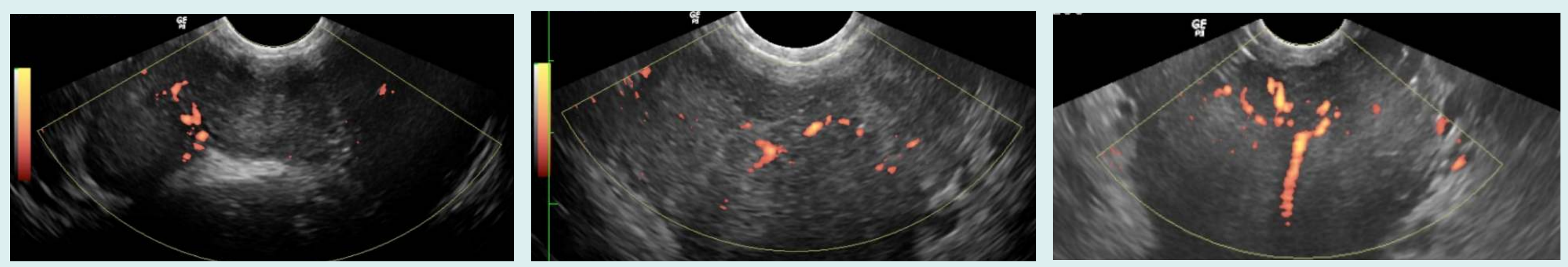

Conclusions: There is a significant relationship between Doppler spectral imagery and the clinical response of external radiation therapy in stage IIB-IVA cervical cancercancer.

Keywords: Cervical cancer; SpectralPulsewave Doppler, Clinical Response

\begin{tabular}{|c|c|c|c|}
\hline \multirow[t]{2}{*}{ Characterictic } & \multicolumn{2}{|c|}{ Clinical Respons } & \multirow[t]{2}{*}{ p) } \\
\hline & $\begin{array}{c}\text { Bad } \\
(n=12)\end{array}$ & $\begin{array}{l}\text { Good } \\
(n=46)\end{array}$ & \\
\hline Age (Year) & 0 & 1 & 313 \\
\hline $26-35$ & 4 & 9 & \\
\hline $36-45$ & 3 & 22 & \\
\hline $46-55$ & 5 & 10 & \\
\hline $\begin{array}{l}56-65 \\
>65\end{array}$ & 0 & 4 & \\
\hline Stage of disease & 7 & 22 & 373 \\
\hline II B & 4 & 2 & \\
\hline III B & 1 & 2 & \\
\hline IV A & & & \\
\hline Histopathology & 2 & 4 & 448 \\
\hline Adeno Ca & 10 & 41 & \\
\hline $\begin{array}{l}\text { Squamous cell carcinoma } \\
\text { Other (clear cell) }\end{array}$ & 0 & 1 & \\
\hline Differentiation & 2 & 3 & 339 \\
\hline Well & 5 & 25 & \\
\hline $\begin{array}{l}\text { Moderately } \\
\text { poorly }\end{array}$ & 2 & 8 & \\
\hline $\begin{array}{l}\text { Tumour type } \\
\text { Bulky } \\
\text { Non Bulky }\end{array}$ & $\begin{array}{l}4 \\
8\end{array}$ & $\begin{array}{l}34 \\
12\end{array}$ & $0.012 *$ \\
\hline $\begin{array}{l}\text { Tumour size before treatment } \\
\text { COP: } 3.5 \mathrm{~cm} \text {, Mean: } 4.76 \\
\text { small }(<3.5 \mathrm{~cm}) \\
\text { big }(\geq 3.5 \mathrm{~cm})\end{array}$ & $\begin{array}{l}3 \\
9\end{array}$ & $\begin{array}{c}6 \\
40\end{array}$ & 271 \\
\hline $\begin{array}{l}\text { Tum o u r volume be fore } \\
\text { treatment } \\
\text { COP: } 46 \mathrm{ml} \text {, Mean: } 70.49 \\
\text { small }(<46 \mathrm{ml}) \\
\text { big ( } \geq 46 \mathrm{ml})\end{array}$ & $\begin{array}{l}8 \\
4\end{array}$ & $\begin{array}{l}16 \\
30\end{array}$ & $0.048^{*}$ \\
\hline
\end{tabular}

\section{Reference:}

1. Alcazar JL, Jurado M, Lopez-Garcia G. Tumor vascularization in cervical cancer by 3-dimensional Pulse Wave Doppler angiography: correlation with tumor characteristics. Int J Gynecol Cancer. 2010:393-7.

2. Huang YF, Cheng YM, Wu YP. Three-dimensional Pulse Wave Doppler ultrasound in cervical carcinoma: monitoring treatment response to radiotherapy. Ultrasound Obstet Gynecol. 2013:84-92.

3. Pirhonen JP, Grenman SA, Bredbacka AB. Effects of external radiotherapy on uterine blood flow in patients with advanced cervical carcinoma assessed by color Doppler ultrasonography. Cancer. 1995:67-71.

4. Chen CA, Cheng WF, Lee CN. Pulse Wave Doppler vascularity index for predicting the response of neoadjuvant chemotherapy in cervical carcinoma. Acta Obstet Gynecol Scand. 2004:591-7. 